\title{
Finite-nuclear-mass calculations of the leading relativistic corrections for atomic $D$ states with all-electron explicitly correlated Gaussian functions
}

\author{
Monika Stanke* \\ Institute of Physics, Faculty of Physics, Astronomy, and Informatics, Nicolaus Copernicus University, ul. Grudziadzka 5, \\ Toruń, PL 87-100, Poland \\ Ludwik Adamowicz ${ }^{\dagger}$ \\ Department of Chemistry and Biochemistry and Department of Physics, University of Arizona, Tucson, Arizona 85721, USA \\ and Interdisciplinary Center for Modern Technologies, Nicolaus Copernicus University, ul. Wileńska 4, Toruń, PL 87-100, Poland
}

(Received 19 June 2019; published 9 October 2019)

\begin{abstract}
An algorithm for calculating the leading relativistic corrections for $D$ states of atoms with an arbitrary number of electrons with all-electron explicitly correlated Gaussian functions is derived and tested in calculations for the helium and beryllium atoms. The finite-nuclear-mass approach used enables us to determine the isotopic shifts of the corrections. The results for interstate transitions for ${ }^{1} D$ states of the helium atom are compared with previous calculations. The results for the beryllium atom are compared with the experimental values, as these are high-accuracy calculations of $D$ states of a four-electron atom performed with the inclusion of the leading relativistic corrections. The calculated and experimental values agree with each other within the experimental error bar.
\end{abstract}

DOI: 10.1103/PhysRevA.100.042503

\section{INTRODUCTION}

Very accurate quantum-mechanical calculations of the ground and excited states of small atoms have always provided the testing ground for new computational methods for atomic calculations. The testing has been possible due to the availability of very accurate gas-phase spectra of these systems. An important group of states for which such high accuracy experimental data are available are $D$ states. For example, in the NIST atomic spectra database [1] among the 182 states of the lithium atom there are ten ${ }^{2} D$ states which correspond to the electron configurations $1 s^{2} n d$, where $n=3,4, \ldots, 12$. For the beryllium atom there are 219 levels listed, 11 of them are ${ }^{1} D$ states and 10 are ${ }^{3} D$ states. The leading electronic configuration in the wave function of the lowest ${ }^{1} D$ state is $1 s^{2} 2 p^{2}$ and in the wave functions of the rest of the states the leading configurations are $1 s^{2} 2 s^{1} n d^{1}$, $n=3,4, \ldots, 12$, respectively. The leading configurations for the ${ }^{3} D$ states are $1 s^{2} n d^{2}, n=3,4, \ldots, 12$. For the boron atom, 66 states with energies below the first ionization energy are listed. There are as many as $38 D$ states with the lowest corresponding to the electron configuration $1 s^{2} 2 s^{1} 2 p^{2}$ and the rest corresponding to the electron configurations $1 s^{2} 2 s^{2} n d^{1}$. The approach developed in this work enables us to calculate the energies of the above-mentioned $D$ states with high accuracy. The high accuracy is achieved by including in the total state energy a very well optimized variational nonrelativistic energy of the atom and the leading relativistic corrections. The calculations are performed using an approach that explicitly

\footnotetext{
*monika@fizyka.umk.pl
}

†ludwik@email.arizona.edu accounts for the finite mass of the atomic nucleus and expanding the spatial part of the wave function in terms of all-electron explicitly correlated Gaussian functions.

While bound states of two- and three-electron atoms have been calculated with very high accuracy using Hylleraastype explicitly correlated functions (see, for example, the calculations concerning the lithium atom performed by Yan and Drake [2], as well as calculations for other two- and three-electron atomic systems [3-7]), the approach involving the Hylleraas functions has not been extended to atoms with four or more than four electrons due to difficulties with calculating the Hamiltonian matrix elements. There is one exception. King et al. [8] calculated the ground states of the beryllium isoelectronic series using a Hylleraas approach with Slater-type basis functions.

Another type of basis function that has been very popular in high-accuracy atomic calculations are explicitly correlated Gaussian functions (ECGs) that exponentially depend on squares of the interelectron distances. The most accurate results for four- and five-electron atoms have been obtained with those functions [9-12]. The largest atomic calculations so far include those of some of the lowest states of the carbon and nitrogen atoms $[13,14]$. ECGs were also used by Pachucki et al. in beryllium [15-18] and boron [19] calculations.

The main advantage of using explicitly correlated Gaussians in atomic calculations is the simplicity of the algorithms for calculating the Hamiltonian and overlap integrals with these functions, which can be analytically evaluated for an arbitrary number of electrons. However, there are some drawbacks of using Gaussians in atomic calculations. These functions do not satisfy the Kato cusp conditions and are decaying too fast at large distances. Thus large ECG basis sets need to be used to achieve satisfactory convergence of 
the energy and other properties. Also, extensive variation optimization of the Gaussian nonlinear parameters needs to be performed to improve the energy convergence [9-12]. In our calculations the optimization is aided by providing the analytically calculated gradient of the energy determined with respect to the Gaussian parameters to the procedure that runs the variational energy minimization. The matrix elements for the energy gradient for the $D$ states, as well as the matrix elements of the Hamiltonian and overlap matrices, were derived and implemented in our 2011 paper [20].

In the approach we use in this work we explicitly account for the finite mass of the nucleus in the variational nonrelativistic calculations, as well as in the calculations of the relativistic corrections. This is done by means of employing a Hamiltonian that explicitly depends on the masses of all particles forming the atom including the mass of the nucleus. The Hamiltonian is obtained by separating out the operator representing the kinetic energy of the center-of-mass motion from the laboratory-frame nonrelativistic Hamiltonian of the system. The finite-nuclear-mass (FNM) approach allows for calculating isotope shifts of the total and transition energies.

As a numerical illustration of the procedure implemented in this work we perform calculations of the relativistic correction of the lowest ten ${ }^{1} D$ states of the helium atom. Eight of these states were calculated before by Drake and Yan [21] and the present results are compared with their results to validate the correctness of the present implementation. We also perform calculation for the lowest two ${ }^{1} D$ states of the beryllium atom. The transition energy calculated as a difference between the total energies of those two states, which include the relativistic corrections, is compared with the experimental value. These are high-accuracy calculations of $D$ states of a four-electron atom performed with the inclusion of the leading relativistic corrections. As the corrections are calculated using nonrelativistic wave functions obtained in FNM calculations and the relativistic operators (obtained by transforming the laboratory-frame operators to an internal coordinate system; the internal coordinate system used in this work is described in the next section), they directly include the so-called recoil effects.

\section{Hamiltonian}

We consider an atom with $N$ particles (i.e., $n=N-1$ electrons and a nucleus). We start with the laboratory-frame nonrelativistic Hamiltonian and we separate out the center-ofmass motion. This is done by introducing an internal Cartesian coordinate system centered at the nucleus. The positions of the electrons in this coordinate system are described by the $\mathbf{r}_{i}$ $(i=1, \ldots, n)$ vectors. The separation of the center-of-mass motion is rigorous and results in the laboratory Hamiltonian becoming a sum of the operator representing the kinetic energy of the center-of-mass motion and the following "internal" Hamiltonian:

$$
\begin{aligned}
\hat{\mathrm{H}}= & -\frac{1}{2}\left(\sum_{i=1}^{n} \frac{1}{\mu_{i}} \nabla_{\mathbf{r}_{i}}^{T} \cdot \nabla_{\mathbf{r}_{i}}+\frac{1}{m_{0}} \sum_{\substack{i, j=1 \\
i \neq j}}^{n} \nabla_{\mathbf{r}_{i}}^{T} \cdot \nabla_{\mathbf{r}_{j}}\right) \\
& +\sum_{i=1}^{n} \frac{q_{0} q_{i}}{r_{i}}+\sum_{i>j=1}^{n} \frac{q_{i} q_{j}}{r_{i j}},
\end{aligned}
$$

where $T$ denotes the matrix-vector transpose; this notation is used throughout the present work, $m_{0}$ is the nucleus mass and $q_{0}$ is its charge, $q_{i}$ are electron charges, and $\mu_{i}=m_{0} m_{i} /\left(m_{0}+m_{i}\right)$ are electron reduced masses $\left(m_{i}, i=\right.$ $2, \ldots, n$, are the electron masses). The Hamiltonian (1) describes the motion of $n$ (pseudo)electrons, whose masses have been changed to the reduced masses, in the central field of the charge of the nucleus. This motion is coupled through the Coulombic interactions: $\sum_{i=1}^{n} \frac{q_{0} q_{i}}{r_{i}}+\sum_{i>j=1}^{n} \frac{q_{i} q_{j}}{r_{i j}}$, where $r_{i j}=\left|\mathbf{r}_{j}-\mathbf{r}_{i}\right|$, and through the mass polarization term, $-\frac{1}{2} \sum_{\substack{i, j=1 \\ i \neq j}}^{n}\left(1 / m_{0}\right) \nabla_{\mathbf{r}_{i}}^{T} \cdot \nabla_{\mathbf{r}_{j}}$. Hamiltonian (1) is used in the present calculations.

\section{BASIS FUNCTIONS}

In this work we consider atomic $D$ states (i.e., $L=2$ states) corresponding to electronic configurations where one or two electrons of the $n$ electron atom is occupying a non-s state. Examples of such states include the above-mentioned $1 s^{2} 2 p^{2}$ and $1 s^{2} 2 s^{1} n d^{1}{ }^{1} D$ states of the beryllium atom. To construct the spatial part of the wave function of an atomic $D$ (with $M_{L}=0$ ) state with a single $d$ electron or two $p$ electrons using explicitly correlated Gaussian functions one needs to use the following functions as products of Gaussian exponentials and Cartesian angular harmonics:

$$
\phi_{k}^{(L=2)}=\left(x_{i_{k}} x_{j_{k}}+y_{j_{k}} y_{i_{k}}-2 z_{i_{k}} z_{j_{k}}\right) \exp \left[-\mathbf{r}^{T}\left(A_{k} \otimes I_{3}\right) \mathbf{r}\right],
$$

where electron labels $i_{k}$ and $j_{k}$, whose value can vary from 1 to $n$, are either equal or not equal to each other. $A_{k}$ in (2) is an $n \times n$ symmetric matrix of the exponential parameters of the Gaussian, $\otimes$ is the Kronecker product, $I_{3}$ is a $3 \times 3$ identity matrix, and $\mathbf{r}$ is a $3 n$ vector that has the form

$$
\mathbf{r}=\left(\begin{array}{c}
\mathbf{r}_{1} \\
\mathbf{r}_{2} \\
\vdots \\
\mathbf{r}_{n}
\end{array}\right)=\left(\begin{array}{c}
x_{1} \\
y_{1} \\
z_{1} \\
\vdots \\
x_{n} \\
y_{n} \\
z_{n}
\end{array}\right) .
$$

We denote $\left(A_{k} \otimes I_{3}\right)$ in (2) as $\mathbf{A}_{k}$. As basis functions (2) are used to expand wave functions of bound atomic states, they need to be square integrable. This only happens if the $\mathbf{A}_{k}$ matrix is positive definite. To make it positive definite we represent it in the Cholesky-factored form as $\mathbf{A}_{k}=\left(L_{k} L_{k}^{T}\right) \otimes$ $I_{3}$, where $L_{k}$ is a $n \times n$ lower triangular matrix. With the $L_{k}$ matrix elements being any real numbers, $\mathbf{A}_{k}$ is positive definite. This is an important feature because it allows us to use the $L_{k}$ matrix elements as the variational optimization parameters, which can be varied without any restrictions in the range from $-\infty$ to $+\infty$. The optimization of these parameters through the variational energy minimization is performed in the present calculations.

The proper permutational symmetry of the wave function in the present calculations is implemented with the use of the spin-free formalism. In this formalism, an appropriate symmetry projector is constructed and applied to the spatial parts of the wave function to impose the desired symmetry properties. The projector, which introduces the desired 
symmetry properties, is constructed using the standard procedure involving Young operators [22-24]. The procedure for generating the permutational symmetry projector was described earlier [25].

For the ${ }^{1} D$ states of the beryllium atom the symmetry projector can be chosen as $P=(1-P 13)(1-P 24)(1+$ $P 12)(1+P 34)$, where $P i j$ permutes the spatial coordinates of the $i$ th and $j$ th electrons. In the calculations of the Hamiltonian and overlap matrix elements, as well as in the operators representing the relativistic corrections, the projector is placed only in the "ket" part of the integrals because the operators are symmetric with respect to permutations of the electron labels. As the projector contains $4 !=24$ terms in the beryllium calculations, each matrix element is a sum of 24 different terms.

We use a general quadratic form, $\mathbf{r}^{T} \mathbf{W}_{k} \mathbf{r}$, in place of $\left(x_{i_{k}} x_{j_{k}}+y_{i_{k}} y_{j_{k}}-2 z_{i_{k}} z_{j_{k}}\right)$ in (2) allowing for a more generalized approach in deriving the matrix elements. With that, our basis functions are

$$
\phi_{k}^{(L=2)}=\left(\mathbf{r}^{T} \mathbf{W}_{k} \mathbf{r}\right) \exp \left[-\mathbf{r}^{T} \mathbf{A}_{k} \mathbf{r}\right]
$$

where $\mathbf{W}_{k}$ is a sparse $3 n \times 3 n$ symmetric matrix that for $i_{k}=j_{k}$ comprises only three nonzero elements: $W_{3\left(i_{k}-1\right)+1,3\left(i_{k}-1\right)+1}=1, \quad W_{3\left(i_{k}-1\right)+2,3\left(i_{k}-1\right)+2}=1, \quad$ and $W_{3\left(i_{k}-1\right)+3,3\left(i_{k}-1\right)+3}=-2$; for $i_{k} \neq j_{k}$ it comprises six nonzero elements: $\quad W_{3\left(i_{k}-1\right)+1,3\left(j_{k}-1\right)+1}=W_{3\left(j_{k}-1\right)+1,3\left(i_{k}-1\right)+1}=$ $\frac{1}{2}, \quad W_{3\left(i_{k}-1\right)+2,3\left(j_{k}-1\right)+2}=W_{3\left(j_{k}-1\right)+2,3\left(i_{k}-1\right)+2}=\frac{1}{2}, \quad$ and $W_{3\left(i_{k}-1\right)+3,3\left(j_{k}-1\right)+3}=W_{3\left(j_{k}-1\right)+3,3\left(i_{k}-1\right)+3}=1$.

\section{RELATIVISTIC OPERATORS}

We consider relativistic corrections of the order of $\alpha^{2}$. The operators representing these corrections, which include the mass-velocity (MV), Darwin (D), orbit-orbit (OO), and spin-spin (SS) corrections, derived in the internal coordinate system with the use of the finite-nuclear-mass approach are (as the states considered in this work are single states, the spin-orbit interaction is zero and is not included in the calculations):

\section{A. Mass-velocity term}

$$
\hat{H}_{\mathrm{MV}}=-\frac{1}{8}\left[\frac{1}{m_{0}^{3}}\left(\sum_{i=1}^{n} \nabla_{\mathbf{r}_{i}}\right)^{4}+\sum_{i=1}^{n} \frac{1}{m_{i}^{3}} \nabla_{\mathbf{r}_{i}}^{4}\right] .
$$

\section{B. Darwin term}

$$
\begin{aligned}
\hat{H}_{\mathrm{D}}= & \frac{\pi}{2} \sum_{i=1}^{n}\left(\frac{4}{3} \frac{1}{m_{0}^{2}}+\frac{1}{m_{i}^{2}}\right) q_{0} q_{i} \delta^{3}\left(\mathbf{r}_{i}\right) \\
& +\frac{\pi}{2} \sum_{i=1}^{n} \sum_{j \neq i}^{n} \frac{1}{m_{i}^{n}} q_{i} q_{j} \delta^{3}\left(\mathbf{r}_{i j}\right) .
\end{aligned}
$$

\section{Orbit-orbit term}

$$
\begin{aligned}
\hat{H}_{\mathrm{OO}}= & -\frac{1}{2} \sum_{i=1}^{n} \sum_{j=1}^{n} \frac{q_{0} q_{j}}{m_{0} m_{j}} \\
& \times\left[\frac{1}{r_{j}} \nabla_{\mathbf{r}_{i}}^{T} \cdot \nabla_{\mathbf{r}_{j}}+\frac{1}{r_{j}^{3}} \mathbf{r}_{j}^{T} \cdot\left(\mathbf{r}_{j}^{T} \cdot \nabla_{\mathbf{r}_{i}}\right) \nabla_{\mathbf{r}_{j}}\right] \\
& +\frac{1}{2} \sum_{i=1}^{n} \sum_{j>i}^{n} \frac{q_{i} q_{j}}{m_{i} m_{j}} \\
& \times\left[\frac{1}{r_{i j}} \nabla_{\mathbf{r}_{i}}^{T} \cdot \nabla_{\mathbf{r}_{j}}+\frac{1}{r_{i j}^{3}} \mathbf{r}_{i j}^{T} \cdot\left(\mathbf{r}_{i j}^{T} \cdot \nabla_{\mathbf{r}_{i}}\right) \nabla_{\mathbf{r}_{j}}\right] .
\end{aligned}
$$

\section{Spin-spin term}

$$
H_{\mathrm{SS}}=-\frac{8 \pi}{3} \sum_{\substack{i, j=1 \\ j>i}}^{4} \frac{q_{i} q_{j}}{m_{i} m_{j}}\left(\mathbf{s}_{i} \cdot \mathbf{s}_{j}\right) \delta\left(\mathbf{r}_{i j}\right),
$$

where $\delta(\mathbf{r})$ is the Dirac delta function and $\mathbf{s}_{i}$ are spin operators for individual electrons. For the states considered in this work $\mathbf{s}_{i} \cdot \mathbf{s}_{j}=-3 / 4$.

The formulas for the matrix elements of the above relativistic operators are presented in the Appendix.

\section{VARIATIONAL CALCULATIONS}

The variational calculations are performed separately and independently for each state and for each state a different basis set is generated. In the calculations the linear expansion coefficients, $c_{k}$, of the wave function in terms of basis functions are obtained in the standard way by solving the secular equation. The nonlinear parameters (i.e., the $L_{k}$ matrix elements) are optimized through the variational minimization of the total nonrelativistic energy. As mentioned, the analytic energy gradient determined with respect to these parameters is used in the minimization [26].

The growing of the basis set for each state is a multistep process. It involves choosing a small starting set of ECGs (for the lowest state this set is generated using an orbital guess obtained using a standard atomic orbital basis set; for a higher state a basis set generated for the next lower state is used as the initial guess; usually a rather small basis set is used as an initial guess). After the initial basis set is optimized the updating of the set starts. It involves addition of small groups of functions, optimizing them, and, after each group of functions is added to the basis set, reoptimizing the whole set. All this is done using the one-function-at-the-time approach. The initial guess for an added function is generated by selecting a set of most contributing functions already included in the basis set, randomly perturbing their nonlinear exponential parameters, and choosing the function which lowers the energy the most. At this stage, the chosen function is added to the basis set and its nonlinear parameters, i.e., the $L_{k}$ matrix elements, are optimized using the procedure that employs the analytic energy 
gradient. Also, at this stage, the $i_{k}$ and $j_{k}$ indices involved in the preexponential angular factor are optimized. This is the only time the optimization of these indices is carried out. After a certain number of functions (100 in the present calculations) is added to the basis set, the entire basis is reoptimized. The reoptimization involves cycling over all functions, one by one, several times and reoptimizing their nonlinear parameters. In the calculations we performed before for other systems it was determined that the above strategy is efficient and numerically stable. The stability of the calculations is also enhanced by checking for any linear dependencies between the basis functions that may appear during the optimization. Such dependencies may lead to numerical inaccuracies and destabilization of the optimization process. If during the optimization of a basis function it becomes linearly dependent with any other function already included in the basis set, the function is reset to what it was before the optimization. The use of the analytic gradient is crucial in making the optimization efficient and in generating very well converged results, i.e., the nonrelativistic energy and the corresponding wave function.

As high-accuracy results of nonrelativistic energies and relativistic corrections obtained with the infinite-nuclear-mass (INM) approach exist in the literature for the helium atom [2], the variational optimization of the nonlinear parameters of the Gaussians is carried out in this work using the INM approach, i.e., the parameters are optimized for ${ }^{\infty} \mathrm{He}$. In the nonlinear-parameter optimization for the beryllium atom the FNM approach is used. Thus, for beryllium, the total nonrelativistic energies obtained in the calculations include the adiabatic and nonadiabatic effects resulting from the finite mass of the nucleus of ${ }^{7} \mathrm{Be}$. The basis sets obtained for ${ }^{7} \mathrm{Be}$ are used to perform the INM energy calculations $\left({ }^{\infty} \mathrm{Be}\right)$ without reoptimization of the nonlinear parameters. As our previous calculations of $S$ and $P$ states of atomic isotopomers have shown, reoptimization of the nonlinear variational parameters is not needed when states of different isotopes are calculated. The adjustment of the linear expansion coefficients of the wave function in terms of the basis functions, $c_{k}$, through rediagonalization of the Hamiltonian is quite sufficient for describing the relatively small changes in the wave function and the energy caused by the change of the nuclear mass. Analogically, for the helium atom, the basis sets obtained in the INM optimizations are used to calculate the nonrelativistic energies and the relativistic corrections for ${ }^{3} \mathrm{He}$ and ${ }^{4} \mathrm{He}$.

To check whether the same applies to $D$ states, such as the ones considered in this work, we took the basis set of 1000 ECGs for the lowest ${ }^{1} D$ state of the helium atom obtained by the variational minimization of the nonrelativistic energy of ${ }^{\infty} \mathrm{He}$ and performed a reoptimization of the ECG nonlinear parameters for the ${ }^{4} \mathrm{He}$ isotope. The ${ }^{4} \mathrm{He}$ energies before and after the reoptimization are equal to -2.05533899479121 and -2.05533899479273 hartree, respectively. As one can see, the improvement is only by about one in the twelve's significant digit after the decimal point. In calculations performed with the double precision, this is close to the expected numerical accuracy of the computation.

\section{RESULTS}

The $D$-state $(L=2)$ atomic code written in Fortran90 that employs the MPI (message passing interface) protocol used in the present calculations has been updated to include the algorithm to calculate the MV, Darwin, OO, and SS relativistic corrections. The code is used in the present calculations.

The first test case concerns the ten lowest ${ }^{1} D$ states of the helium atom. The basis sets for the lowest two states is grown to the size of 1600 functions, for the third to the size of 1800 functions, and for the remaining states to the size of 2000 function. As mentioned, the basis set optimizations are performed using the internal Hamiltonian with an infinite nuclear mass and the calculations of the energies for the ${ }^{3} \mathrm{He}$ and ${ }^{4} \mathrm{He}$ isotopes are performed without reoptimization of the basis set exponential parameters. The convergence of the nonrelativistic energies of the ten states with the number of the basis functions is shown in Table I. The ${ }^{\infty} \mathrm{He}$ energies are compared with the energies obtained by Drake using the Hylleraas type functions [27]. As the comparison shows, for some lowest states the present energies agree with the energies of Drake to 13 digits. After that the agreement gets progressively worse, indicating that the basis sets need to be extended beyond 2000 functions. It is worth noticing that in the present calculations two more states than considered by Drake are determined (states $11{ }^{1} D$ and $12{ }^{1} D$ ).

The MV, D, and OO relativistic corrections calculated for each considered state with the largest basis set are shown in Table II. The results obtained for ${ }^{\infty} \mathrm{He}$ are compared with the results of Drake and Yan [21]. As one can see the agreement is very good particularly for lower states. It is particularly interesting to examine the $\mathrm{OO}$ correction. The algorithm for calculating this correction contains two types of terms. The first type comprising two-electron contributions does not depend on the mass of the nucleus. The second type includes terms dependent on the inverse of the nuclear mass. As the level of excitation increases and one of the electrons becomes excited to an increasingly more diffuse state and, thus, becomes separated from the first electron, which remains in the core $1 s$ state, the $\mathrm{OO}$ correction calculated for ${ }^{\infty} \mathrm{He}$ converges to zero. But, when the calculation is performed for either ${ }^{3} \mathrm{He}$ or ${ }^{4} \mathrm{He}$, the terms dependent on the nuclear mass dominate the correction. These terms describe the magnetic interaction of the orbiting electrons with the orbiting nucleus. The orbiting motion occurs around the center of mass of the atom. For an infinite nuclear mass this interaction is zero. Now, as the atom becomes excited to an increasingly higher Rydberg state and one of the electrons (the $d$ electron) almost departs from the atom, the interaction should be converging to the $\mathrm{OO}$ finite-nuclear-mass interaction in the ground ${ }^{2} S$ state of the $\mathrm{He}^{+}$ion, which is zero. The results for the ${ }^{4} \mathrm{He}^{+}$and ${ }^{3} \mathrm{He}^{+}$ions are shown in Table II and, as one can see, the expected convergence trend is indeed occurring. The agreement between the present values of the MV, D, and OO relativistic corrections calculated for ${ }^{\infty} \mathrm{He}$ with the results obtained by Drake and Yan [21] and the correct asymptotic convergence of the corrections calculated for ${ }^{4} \mathrm{He}$ and ${ }^{3} \mathrm{He}$ to the corresponding values calculated for the ${ }^{4} \mathrm{He}^{+}$and ${ }^{3} \mathrm{He}^{+}$ 


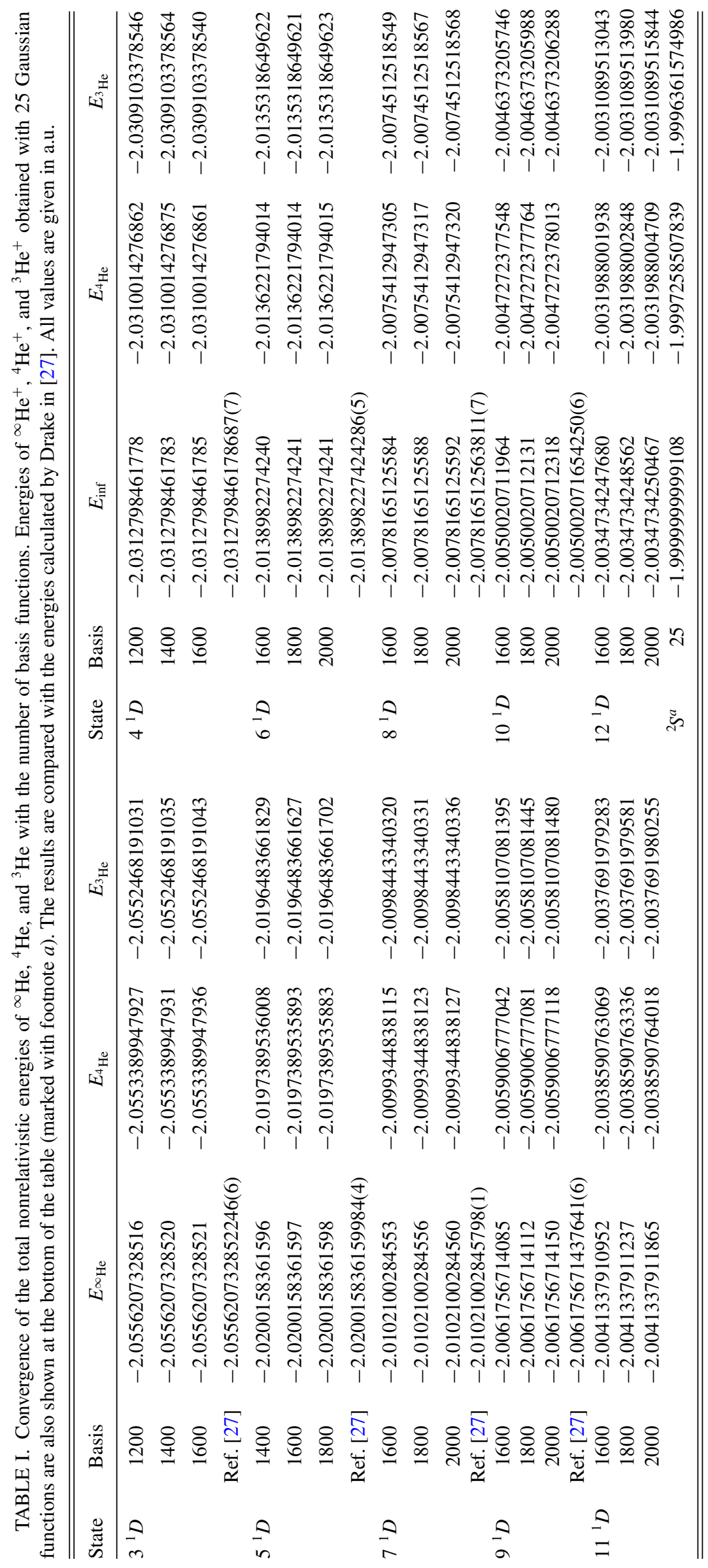




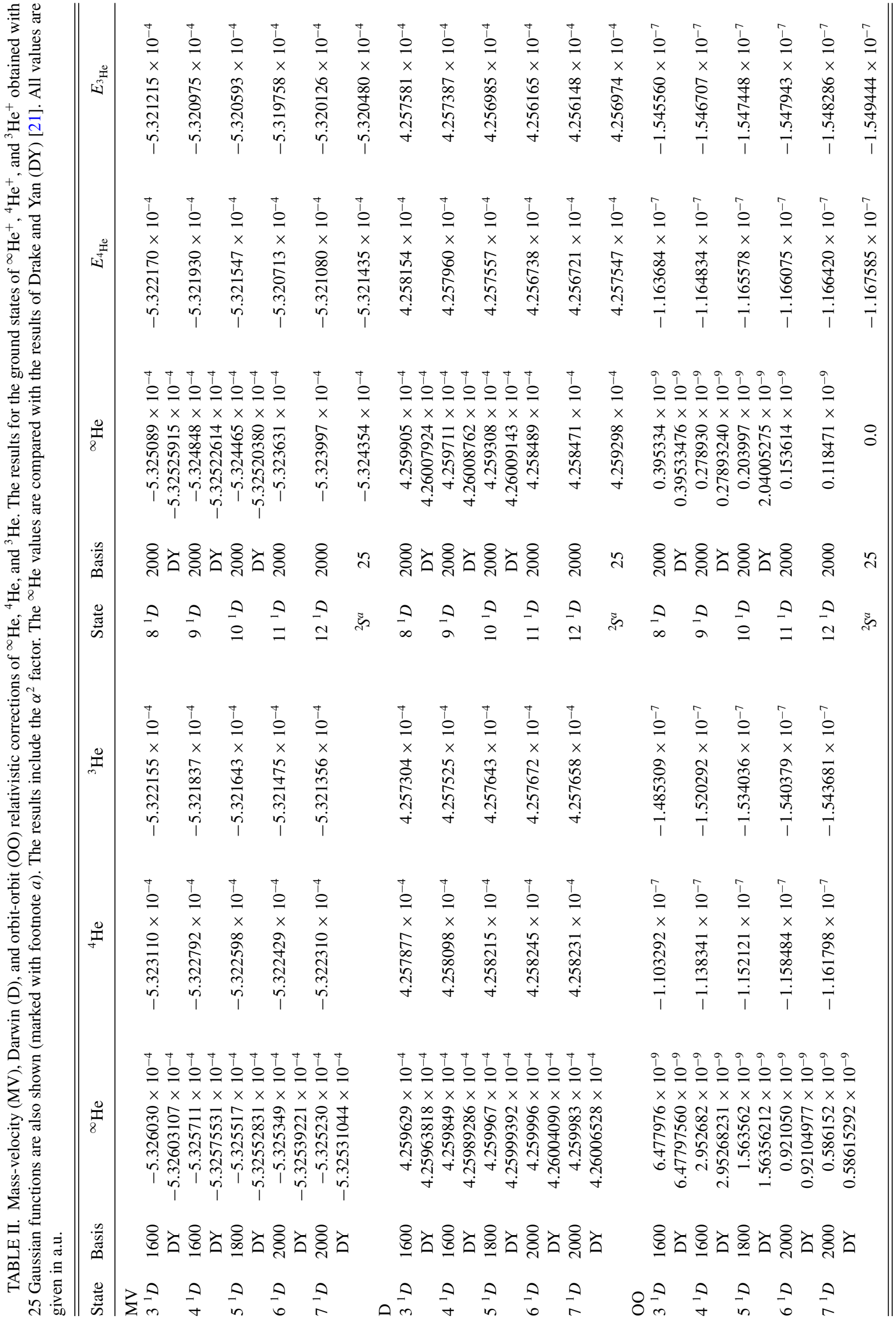


TABLE III. Convergence of the nonrelativistic energy $\left(E_{\mathrm{nr}}\right)$, mass-velocity (MV), Darwin (D), orbit-orbit (OO), and spin-spin (SS) relativistic corrections of the two lowest ${ }^{1} D$ states $\left(3^{1} D\right.$ and $\left.4{ }^{1} D\right)$ of ${ }^{9} \mathrm{Be}$. The results for ${ }^{\infty} \mathrm{Be}$ are also shown. The MV, D, OO, and SS corrections include the $\alpha^{2}$ factor. All values are given in a.u.

\begin{tabular}{|c|c|c|c|c|c|c|c|}
\hline System & State & Basis & $E_{\mathrm{nr}}$ & MV & $\mathrm{D}$ & $\mathrm{OO}$ & SS \\
\hline${ }^{9} \mathrm{Be}$ & $3{ }^{1} D$ & $\begin{array}{c}2100 \\
3000 \\
3900 \\
10500 \\
11400 \\
12300\end{array}$ & $\begin{array}{l}-14.407349580 \\
-14.407350719 \\
-14.407351053 \\
-14.407351368 \\
-14.407351371 \\
-14.407351373\end{array}$ & $\begin{array}{l}-0.014033610 \\
-0.014033923 \\
-0.014035800\end{array}$ & $\begin{array}{l}0.011295071 \\
0.011296120 \\
0.011297961\end{array}$ & $\begin{array}{l}-0.000038177 \\
-0.000038177 \\
-0.000038177\end{array}$ & $\begin{array}{l}0.000512155 \\
0.000511957 \\
0.000511952\end{array}$ \\
\hline${ }^{\infty} \mathrm{Be}$ & $3{ }^{1} D$ & $\begin{array}{c}3900 \\
12300\end{array}$ & $\begin{array}{l}-14.408236961 \\
-14.408237282\end{array}$ & -0.014039343 & 0.011300109 & -0.000036806 & 0.000512046 \\
\hline${ }^{9} \mathrm{Be}$ & $4{ }^{1} D$ & $\begin{array}{c}2100 \\
3000 \\
3900 \\
10500 \\
11400 \\
12300\end{array}$ & $\begin{array}{l}-14.372923384 \\
-14.372924394 \\
-14.372924677 \\
-14.372924942 \\
-14.372924945 \\
-14.372924948\end{array}$ & $\begin{array}{l}-0.014211577 \\
-0.014215861 \\
-0.014215932\end{array}$ & $\begin{array}{l}0.011423474 \\
0.011427686 \\
0.011428019\end{array}$ & $\begin{array}{l}-0.000046760 \\
-0.000046760 \\
-0.000046760\end{array}$ & $\begin{array}{l}0.000525309 \\
0.000525178 \\
0.000525099\end{array}$ \\
\hline${ }^{\infty} \mathrm{Be}$ & $4{ }^{1} D$ & $\begin{array}{c}3900 \\
12300\end{array}$ & $\begin{array}{l}-14.373824331 \\
-14.373824602\end{array}$ & -0.014219326 & 0.011429766 & -0.000045361 & 0.000525184 \\
\hline
\end{tabular}

ions validates the algorithms derived and implemented in the present work.

The next set of calculations is performed for the lowest two ${ }^{1} D$ states of the beryllium atom. These are high-accuracy calculations for these states. The ECG basis sets for each state are grown to the level of 12300 functions and the optimizations of the Gaussian nonlinear parameters are carried out for the ${ }^{9} \mathrm{Be}$ isotope. After the 12300-ECGs basis set is generated for each state, the energy for ${ }^{\infty} \mathrm{Be}$ is calculated without reoptimization of the exponential parameters. As mentioned, only the linear expansion parameters of the wave functions of the two states are reevaluated by solving the standard secular equation with the internal Hamiltonian, where the nuclear mass is set to infinity. With 12300 ECGs in the basis set the total nonrelativistic energies of the two states are converged to 10-11 digits. The results are shown in Table III.

Unfortunately, our present computational resources do not allow for calculating the relativistic corrections with the full 12300-ECGs basis sets. Even with extensive optimization

TABLE IV. Calculation of the $3{ }^{1} D \rightarrow 4{ }^{1} D$ transition energy and its comparison with the experimental value [1]. The transition energy is calculated for ${ }^{\infty} \mathrm{Be}$ and ${ }^{9} \mathrm{Be}$ without the relativistic corrections $\left(\Delta E_{\mathrm{nr}}\right)$ using the total nonrelativistic energies obtained with 12300 ECGs, and with the addition of the relativistic MV, D, OO, and SS corrections $\left(\Delta E_{\text {rel }}\right)$ calculated with 1200,2100 , and 3000 ECGs. All values are given in $\mathrm{cm}^{-1}$.

\begin{tabular}{lccc}
\hline \hline & \multicolumn{3}{c}{ Basis } \\
\hline${ }^{\infty} \mathrm{Be}$ & $\Delta E_{\mathrm{nr}}$ & 12300 & 7552.65 \\
${ }^{\infty} \mathrm{Be}$ & $\Delta E_{\mathrm{rel}}$ & $12300+3900$ & 7542.68 \\
${ }^{9} \mathrm{Be}$ & $\Delta E_{\mathrm{nr}}$ & 12300 & 7555.78 \\
& $\Delta E_{\mathrm{rel}}$ & $12300+2100$ & 7545.91 \\
& & $12300+3000$ & 7545.75 \\
& & $12300+3900$ & $7545.82(7)$ \\
Experiment [1] & & & $7545.88(20)$ \\
\hline \hline
\end{tabular}

of the algorithms and with the implementation of a parallel approach in the computer code using the MPI protocol, the practical limit for the basis set size for which the calculations can be performed in our laboratory is lower than the number of functions used to calculate the nonrelativistic energies. Thus, in addition to the nonrelativistic energies obtained with 10500 , 11400, and 12300 ECGs, the energies and the relativistic corrections obtained with 2100, 3000, and 3900 ECGs are shown in Table III. Both sets of results correspond to the ${ }^{9} \mathrm{Be}$ isotope. For ${ }^{\infty} \mathrm{Be}$ only the results obtained with 3900 and 12300 ECGs are shown. As one can see, with 3900 ECGs eight digits in the total nonrelativistic energies are practically already converged. However, more importantly, the relativistic corrections are also very well converged for both states.

Finally, the results presented in Table III are used to calculate the $3{ }^{1} \mathrm{D} \rightarrow 4^{1} \mathrm{D}$ transition energy. The energy is calculated by taking the difference between the nonrelativistic energies calculated for the two states with 12300 ECGs and adding to it the differences of the relativistic energies calculated with 2100, 3000, and 3900 ECGs. The obtained values are compared with the experimental transition energy in Table IV. As one can see, the inclusion of the relativistic corrections significantly improves the agreement between the calculated transition energy and the experimental value. Our best calculated transition energy of $7545.82 \mathrm{~cm}^{-1}$ of ${ }^{9} \mathrm{Be}$ agrees with the experimental result of $7545.88 \mathrm{~cm}^{-1}$ obtained by subtracting the energies of the $3{ }^{1} \mathrm{D}$ and $4{ }^{1} \mathrm{D}$ levels [1] to within $0.06 \mathrm{~cm}^{-1}$. This is significantly less than the experimental error bar of $0.2 \mathrm{~cm}^{-1}$. These are by far the most accurate calculations ever performed for $D$ states of a four electron atom. The contribution from the leading relativistic corrections to the ${ }^{1} D \rightarrow 4^{1} D$ transition energy is nearly $10 \mathrm{~cm}^{-1}$.

\section{SUMMARY}

In summary, algorithms for calculating the leading spinindependent relativistic corrections for atomic $D$ states are de- 
rived and implemented using Fortran90 and the MPI protocol. The corrections are calculated using the nonrelativistic wave functions obtained in variational calculations performed with an approach where the Hamiltonian describing the internal state of the atom explicitly depends on the finite mass of the nucleus. The spatial parts of the wave functions are expanded in terms of explicitly correlated Gaussian functions. Thorough variational optimization of the exponential parameters of the Gaussians performed with a procedure that employs the analytical energy gradient and the use of large basis sets enables us to obtain very accurate results. The implemented algorithms are first tested in calculations of the ten lowest ${ }^{1} D$ states of the helium atom. The calculations are performed for finite and infinite masses of the helium nucleus. The infinitenuclear-mass results are compared with literature results and the finite-nuclear-mass results are tested for their convergence to the results obtained for ${ }^{3} \mathrm{He}^{+}$and ${ }^{4} \mathrm{He}^{+}$ions. We also perform calculations for the two lowest ${ }^{1} D$ states of the beryllium atom to test if the inclusion of the relativistic corrections significantly improves the agreement between the calculated and experimental results for the $3{ }^{1} \mathrm{D} \rightarrow 4{ }^{1} \mathrm{D}$ transition energy. The calculated and experimental values of the transition energy agree with each other within the experimental error bar. A significant improvement is indeed achieved. Calculations of higher bound $D$ states of beryllium, as well as of other small atomic systems, are forthcoming.

\section{ACKNOWLEDGMENTS}

This work of M.S. has been supported by the Polish National Science Centre, Grant No. DEC$2013 / 10 / E / S T 4 / 00033$. It has also been supported by a grant from the National Science Foundation, Grant No. 1856702. The authors are grateful to the University of Arizona Research Computing for providing computational resources for this work.

\section{APPENDIX}

The general form of the basis functions for describing the $L=2$ states used in this work is

$$
\phi_{k}^{(L=2)}=\left(\mathbf{r}^{T} \mathbf{W}_{k} \mathbf{r}\right) \exp \left[-\mathbf{r}^{T} \mathbf{A}_{k} \mathbf{r}\right]=\left(\mathbf{r}^{T} \mathbf{W}_{k} \mathbf{r}\right) \varphi_{k}^{(L=0)} \equiv\left(\mathbf{r}^{T} \mathbf{W}_{k} \mathbf{r}\right) \varphi_{k}
$$

$\hat{H}_{\mathrm{MV}}$. The matrix elements that need to be calculated are

$$
\left\langle\phi_{k}^{(L=2)}\left|\hat{H}_{\mathrm{MV}}\right| \phi_{l}^{(L=2)}\right\rangle=-\frac{1}{8}\left(\frac{1}{m_{0}^{3}}\left\langle\nabla_{\mathbf{r}}^{T} \mathbf{J} \nabla_{\mathbf{r}} \phi_{k}^{(L=2)} \mid \nabla_{\mathbf{r}}^{T} \mathbf{J} \nabla_{\mathbf{r}} \phi_{l}^{(L=2)}\right\rangle+\sum_{i=1}^{n} \frac{1}{m_{i+1}^{3}}\left\langle\nabla_{\mathbf{r}}^{T} \mathbf{J}_{i i} \nabla_{\mathbf{r}} \phi_{k}^{(L=2)} \mid \nabla_{\mathbf{r}}^{T} \mathbf{J}_{i i} \nabla_{\mathbf{r}} \phi_{l}^{(L=2)}\right\rangle\right),
$$

where square matrix $\mathbf{J}$ (with no indices) is a matrix whose all elements are equal one, $\mathbf{J}_{\alpha \beta}=1$. Square matrix $\mathbf{J}_{i j}$ is defined as

$$
\mathbf{J}_{i j}= \begin{cases}\mathbf{E}_{i i}, & \text { if } i=j, \\ \mathbf{E}_{i i}+\mathbf{E}_{j j}-\mathbf{E}_{i j}-\mathbf{E}_{j i}, & \text { if } i \neq j .\end{cases}
$$

Only the following one type of integral appears in the expression for the $\hat{H}_{\mathrm{MV}}$ matrix element: $\left\langle\nabla_{\mathbf{r}}^{T} \mathbf{D} \nabla_{\mathbf{r}} \phi_{k} \mid \nabla_{\mathbf{r}}^{T} \mathbf{D} \nabla_{\mathbf{r}} \phi_{l}\right\rangle$, where $\mathbf{D}$ is either $\mathbf{J}$ or $\mathbf{J}_{i i}$. In order to calculate the expectation value of $\hat{H}_{\mathrm{MV}}$, the following result is used in bra and ket:

$$
\nabla_{\mathbf{r}}^{T} \mathbf{D} \nabla_{\mathbf{r}} \phi^{(L=2)}=-2 \operatorname{Tr}[\mathbf{A D}]\left(\mathbf{r}^{T} \mathbf{W r}\right) \varphi+4\left(\mathbf{r}^{T} \mathbf{A}_{l} \mathbf{D} \mathbf{A r}\right)\left(\mathbf{r}^{T} \mathbf{W r}\right) \varphi+\operatorname{Tr}[\underline{\mathbf{W D}}] \varphi-2\left(\mathbf{r}^{T} \mathbf{A} \mathbf{D} \underline{\mathbf{W}}\right) \varphi-2\left(\mathbf{r}^{T} \underline{\mathbf{W}} \mathbf{D} \mathbf{A r}\right) \varphi,
$$

where $\underline{\mathbf{W}}=\mathbf{W}^{T}+\mathbf{W}$ and where $\phi^{(L=2)}$ is either $\phi_{k}^{(L=2)}$ or $\phi_{l}^{(L=2)}$.

$\hat{H}_{\mathrm{D}}$. To calculate the expectation value of the Darwin operator we need the matrix elements of the following Dirac delta function:

$$
\left\langle\phi_{k}^{(L=2)}\left|\delta\left(\left(a \otimes I_{3}\right)^{T} \mathbf{r}\right)\right| \phi_{l}^{(L=2)}\right\rangle=\left\langle\varphi_{k}\left|\left(\mathbf{r}^{T} \mathbf{W}_{k} \mathbf{r}\right) \delta\left(\left(a \otimes I_{3}\right)^{T} \mathbf{r}\right)\left(\mathbf{r}^{T} \mathbf{W}_{l} \mathbf{r}\right)\right| \varphi_{l}\right\rangle,
$$

where $a=e^{i} a a^{T} \otimes I_{3}^{T}=\mathbf{J}_{i i}$ and $a=e^{i}-e^{j} a a^{T} \otimes I_{3}^{T}=\mathbf{J}_{i j}$. Using the following representation of the delta function:

$$
\delta\left(\left(a \otimes I_{3}\right)^{T} \mathbf{r}\right)=\lim _{\beta \rightarrow \infty}\left(\frac{\beta}{\pi}\right)^{3 / 2} \exp \left[-\beta \mathbf{r}^{T}\left(a a^{T} \otimes I_{3}\right) \mathbf{r}\right],
$$

we can express (A5) as follows:

$$
\begin{aligned}
& \left\langle\phi_{k}^{(L=2)}\left|\delta\left(\left(a \otimes I_{3}\right)^{T} \mathbf{r}\right)\right| \phi_{l}^{(L=2)}\right\rangle \\
& =\lim _{\beta \rightarrow \infty}\left(\frac{\beta}{\pi}\right)^{3 / 2}\left\langle\varphi_{k}\left|\left(\mathbf{r}^{T} \mathbf{W}_{k} \mathbf{r}\right) \exp \left[-\beta \mathbf{r}^{T}\left(a a^{T} \otimes I_{3}\right) \mathbf{r}\right]\left(\mathbf{r}^{T} \mathbf{W}_{l} \mathbf{r}\right)\right| \varphi_{l}\right\rangle \\
& =\lim _{\beta \rightarrow \infty}\left(\frac{\beta}{\pi}\right)^{3 / 2} \frac{\pi^{3 n / 2}}{\left|A_{k l}+\beta a a^{T}\right|^{3 / 2}} \frac{1}{2}\left\{\frac{1}{2} \operatorname{Tr}\left[\left[\mathbf{A}_{k l}+\beta\left(a a^{T} \otimes I_{3}\right)\right]^{-1} \mathbf{W}_{l}\right] \operatorname{Tr}\left[\left[\mathbf{A}_{k l}+\beta\left(a a^{T} \otimes I_{3}\right)\right]^{-1} \mathbf{W}_{k}\right]\right. \\
& \left.\quad+\operatorname{Tr}\left[\left[\mathbf{A}_{k l}+\beta\left(a a^{T} \otimes I_{3}\right)\right]^{-1} \mathbf{W}_{l}\left[\mathbf{A}_{k l}+\beta\left(a a^{T} \otimes I_{3}\right)\right]^{-1} \mathbf{W}_{k}\right]\right\}
\end{aligned}
$$


where we used the following mathematical "trick":

$$
\begin{aligned}
\left\langle\varphi_{k}\left|\left(\mathbf{r}^{T} \mathbf{W}_{k} \mathbf{r}\right)\left(\mathbf{r}^{T} \mathbf{W}_{l} \mathbf{r}\right)\right| \varphi_{l}\right\rangle & =\left.\frac{\partial}{\partial \alpha_{k}} \frac{\partial}{\partial \alpha_{l}} \int \mathrm{d} \mathbf{r} \exp \left[-\mathbf{r}^{T}\left(\mathbf{A}_{k l}+\alpha_{k} \mathbf{W}_{k}+\alpha_{l} \mathbf{W}_{l}\right) \mathbf{r}\right]\right|_{\alpha_{k}=\alpha_{l}=0} \\
& =\frac{\pi^{3 n / 2}}{\left|A_{k l}\right|^{3 / 2}} \frac{1}{2}\left\{\frac{1}{2} \operatorname{Tr}\left[\mathbf{A}_{k l}^{-1} \mathbf{W}_{l}\right] \operatorname{Tr}\left[\mathbf{A}_{k l}^{-1} \mathbf{W}_{k}\right]+\operatorname{Tr}\left[\mathbf{A}_{k l}^{-1} \mathbf{W}_{l} \mathbf{A}_{k l}^{-1} \mathbf{W}_{k}\right]\right\} \\
\left\langle\varphi_{k}\left|\left(\mathbf{r}^{T} \mathbf{W}_{k} \mathbf{r}\right)\left(\mathbf{r}^{T} \mathbf{W}_{l} \mathbf{r}\right) \exp \left[-\mathbf{r}^{T} \mathbf{X r}\right]\right| \varphi_{l}\right\rangle= & \left.\frac{\partial}{\partial \alpha_{k}} \frac{\partial}{\partial \alpha_{l}} \int \mathrm{d} \mathbf{r} \exp \left[-\mathbf{r}^{T}\left(\mathbf{A}_{k l}+\mathbf{X}+\alpha_{k} \mathbf{W}_{k}+\alpha_{l} \mathbf{W}_{l}\right) \mathbf{r}\right]\right|_{\alpha_{k}=\alpha_{l}=0} \\
= & \frac{\pi^{3 n / 2}}{\left|A_{k l}+X\right|^{3 / 2}} \frac{1}{2}\left\{\frac{1}{2} \operatorname{Tr}\left[\left(\mathbf{A}_{k l}+\mathbf{X}\right)^{-1} \mathbf{W}_{l}\right] \operatorname{Tr}\left[\left(\mathbf{A}_{k l}+\mathbf{X}\right)^{-1} \mathbf{W}_{k}\right]\right. \\
& \left.+\operatorname{Tr}\left[\left(\mathbf{A}_{k l}+\mathbf{X}\right)^{-1} \mathbf{W}_{l}\left(\mathbf{A}_{k l}+\mathbf{X}\right)^{-1} \mathbf{W}_{k}\right]\right\}
\end{aligned}
$$

$\hat{H}_{\mathrm{OO}}$. The matrix element of the $\hat{H}_{\mathrm{OO}}$ operator is calculated analogically as described in our previous paper [28]. In the derivation the following partial derivatives are used:

$$
\begin{aligned}
& \partial^{\alpha} \partial_{\beta} \phi_{k}^{(L=2)}=\left\{-2\left(\mathbf{A}_{k}\right)_{\beta}^{\alpha}\left(\mathbf{r}^{T} \mathbf{W}_{k} \mathbf{r}\right)+\left(\underline{\mathbf{W}}_{k}\right)_{\beta}^{\alpha}+4\left(\mathbf{r} \mathbf{A}_{k}\right)^{\alpha}\left(\mathbf{A}_{k} \mathbf{r}\right)_{\beta}\left(\mathbf{r}^{T} \mathbf{W}_{k} \mathbf{r}\right)-\left(\mathbf{r} \mathbf{A}_{k}\right)^{\alpha}\left(\underline{\mathbf{W}_{\mathbf{k}}} \mathbf{r}\right)_{\beta}-\left(\mathbf{r} \underline{\mathbf{W}}_{k}\right)^{\alpha}\left(\mathbf{A}_{k} \mathbf{r}\right)_{\beta}\right\} \varphi_{k}, \\
& \partial^{\rho} \partial_{\alpha} \partial_{\beta} \phi_{k}^{(L=2)}=\left[+4\left(\mathbf{A}_{k}\right)_{\alpha}{ }^{\rho}\left(\mathbf{A}_{k} \mathbf{r}\right)_{\beta}\left(\mathbf{r}^{T} \mathbf{W}_{k} \mathbf{r}\right)+4\left(\mathbf{A}_{k} \mathbf{r}\right)_{\alpha}\left(\mathbf{A}_{k}\right)_{\beta}{ }^{\rho}\left(\mathbf{r}^{T} \mathbf{W}_{k} \mathbf{r}\right)-2\left(\mathbf{A}_{k}\right)_{\alpha}{ }^{\rho}\left(\underline{\mathbf{W}_{\mathbf{k}}} \mathbf{r}\right)_{\beta}-2\left(\mathbf{A}_{k} \mathbf{r}\right)_{\alpha}\left(\underline{\mathbf{W}_{\mathbf{k}}}\right)_{\beta}{ }^{\rho}\right. \\
& -2\left(\underline{\mathbf{W}}_{k}\right)_{\alpha}{ }^{\rho}\left(\mathbf{A}_{k} \mathbf{r}\right)_{\beta}-2\left(\underline{\mathbf{W}}_{k} \mathbf{r}\right)_{\alpha}\left(\mathbf{A}_{k}\right)_{\beta}{ }^{\rho} \\
& +4\left(\mathbf{A}_{k}\right)_{\alpha \beta}\left(\mathbf{r} \mathbf{A}_{k}\right)^{\rho}\left(\mathbf{r}^{T} \mathbf{W}_{k} \mathbf{r}\right)-2\left(\underline{\mathbf{W}}_{k}\right)_{\alpha \beta}\left(\mathbf{r} \mathbf{A}_{k}\right)^{\rho}-8\left(\mathbf{A}_{k} \mathbf{r}\right)_{\alpha}\left(\mathbf{A}_{k} \mathbf{r}\right)_{\beta}\left(\mathbf{r} \mathbf{A}_{k}\right)^{\rho}\left(\mathbf{r}^{T} \mathbf{W}_{k} \mathbf{r}\right)+4\left(\mathbf{A}_{k} \mathbf{r}\right)_{\alpha}\left(\underline{\mathbf{W}}_{\mathbf{k}} \mathbf{r}\right)_{\beta}\left(\mathbf{r} \mathbf{A}_{k}\right)^{\rho} \\
& \left.+4\left(\underline{\mathbf{W}}_{k} \mathbf{r}\right)_{\alpha}\left(\mathbf{A}_{k} \mathbf{r}\right)_{\beta}\left(\mathbf{r} \mathbf{A}_{k}\right)^{\rho}-2\left(\mathbf{A}_{k}\right)_{\alpha \beta}\left(\mathbf{r} \underline{\mathbf{W}}_{k}\right)^{\rho}+4\left(\mathbf{A}_{k} \mathbf{r}\right)_{\alpha}\left(\mathbf{A}_{k} \mathbf{r}\right)_{\beta}\left(\mathbf{r} \underline{\mathbf{W}}_{k}\right)^{\rho}\right] \varphi_{k} \text {. }
\end{aligned}
$$

More details concerning the calculations of the elementary integrals contributing to the above-described matrix elements can be found in the Supplemental Material [29].

[1] A. E. Kramida, Y. Ralchenko, J. Reader, and NIST ASD Team, NIST Atomic Spectra Database, ver. 5.6 (online) (National Institute of Standards and Technology, Gaithersburg, MD, 2018), http://physics.nist.gov/asd.

[2] L. M. Wang, C. Li, Z.-C. Yan, and G. W. F. Drake, Phys. Rev. A 95, 032504 (2017).

[3] P. J. Pelzl, G. J. Smethells, and F. W. King, Phys. Rev. E 65, 036707 (2002).

[4] D. M. Feldmann, P. J. Pelzl, and F. W. King, J. Math. Phys. 39, 6262 (1998).

[5] Z.-C. Yan, M. Tambasco, and G. W. F. Drake, Phys. Rev. A 57, 1652 (1998).

[6] Z.-C. Yan, W. Nörtershäuser, and G. W. F. Drake, Phys. Rev. Lett. 100, 243002 (2008).

[7] M. Puchalski and K. Pachucki, Phys. Rev. A 73, 022503 (2006).

[8] F. W. King, D. Quicker, and J. Langer, J. Chem. Phys. 134, 124114 (2011).

[9] M. Stanke, D. Kedziera, S. Bubin, and L. Adamowicz, Phys. Rev. Lett. 99, 043001 (2007).

[10] S. Bubin, J. Komasa, M. Stanke, and L. Adamowicz, Phys. Rev. A 81, 052504 (2010).

[11] S. Bubin, J. Komasa, M. Stanke, and L. Adamowicz, J. Chem. Phys. 132, 114109 (2010).
[12] S. Bubin, M. Stanke, and L. Adamowicz, J. Chem. Phys. 131, 044128 (2009).

[13] K. L. Sharkey, M. Pavanello, S. Bubin, and L. Adamowicz, Phys. Rev. A 80, 062510 (2009).

[14] K. L. Sharkey and L. Adamowicz, J. Chem. Phys. 140, 174112 (2014).

[15] K. Pachucki and J. Komasa, Phys. Rev. Lett. 92, 213001 (2006).

[16] K. Pachucki and J. Komasa, Phys. Rev. A 73, 052502 (2006).

[17] M. Puchalski, J. Komasa, and K. Pachucki, Phys. Rev. A 87, 030502(R) (2013).

[18] M. Puchalski, K. Pachucki, and J. Komasa, Phys. Rev. A 89, 012506 (2014).

[19] M. Puchalski, J. Komasa, and K. Pachucki, Phys. Rev. A 92, 062501 (2015).

[20] K. L. Sharkey, S. Bubin, and L. Adamowicz, J. Chem. Phys. 134, 044120 (2011).

[21] G. W. F. Drake and Z.-Ch. Yan, Phys. Rev. A 46, 2378 (1992).

[22] F. A. Matsen and R. Pauncz, The Unitary Group in Quantum Chemistry (Elsevier, Amsterdam, 1986).

[23] R. Pauncz, Spin Eigenfunctions (Plenum, New York, 1979).

[24] M. Hamermesh, Group Theory and Its Application to Physical Problems (Addison-Wesley, Reading, MA, 1962).

[25] S. Bubin, M. Cafiero, and L. Adamowicz, Adv. Chem. Phys. 131, 377 (2005). 
[26] S. Bubin, M. Pavanello, W.-C. Tung, K. L. Sharkey, and L. Adamowicz, Chem. Rev. 113, 36 (2013).

[27] G. W. F. Drake, in Atomic, Molecular and Optical Physics Handbook, edited by G. W. F. Drake (AIP Press, New York, 1996), pp. 154-171.
[28] M. Stanke, A. Bralin, S. Bubin, and L. Adamowicz, Phys. Rev. A. 97, 012513 (2018).

[29] See Supplemental Material at http://link.aps.org/supplemental/ 10.1103/PhysRevA.100.042503 for detailed evaluation of the integrals involved in calculating the matrix elements of the MV, $\mathrm{D}, \mathrm{OO}$, and SS relativistic operators. 